ORIGINAL ARTICLE

\title{
Canalicular immunostaining of aminopeptidase N (CD13) as a diagnostic marker for hepatocellular carcinoma
}

\author{
C Röcken, J Licht, A Roessner, S Carl-McGrath
}

J Clin Pathol 2005;58:1069-1075. doi: 10.1136/jcp.2005.026328

See end of article for authors' affiliations

.....................

Correspondence to: Professor C Röcken Department of Pathology, Otto-von-GuerickeUniversity, Leipziger Str. 44, D-39120 Magdeburg, Germany; christoph. roecken@medizin.unimagdeburg.de

Accepted for publication 3 February 2005
Background: Aminopeptidase N (CD13) is expressed in normal and neoplastic liver tissue, where it exhibits a characteristic canalicular pattern (CD13 $\left.{ }^{\text {can }}\right)$, similar to that seen for CD10 and when antibodies crossreact with biliary glycoprotein I ( $\mathrm{p}$-CEA).

Aim: To compare the putative diagnostic use of $C D 13^{\text {can }}$ in differentiating between hepatocellular (HCC) and non-hepatocellular carcinomas metastatic to the liver (non-HCC).

Methods: A retrospective study comparing $53 \mathrm{HCC}$ specimens with 32 non-HCC specimens. Immunostaining was performed with HepParl and antibodies directed against CD10, CD13, p-CEA, and $\alpha$ fetoprotein (AFP).

Results: In the HCC group, a canalicular staining pattern was found for CD13, p-CEA, and CD10 in 51, 43 , and 33 specimens, respectively. HepParl was positive in 29 and AFP in 17 HCC specimens. In the non-HCC group, canalicular immunostaining for CD10 and p-CEA was confined to non-neoplastic liver tissue. One poorly differentiated cholangiocarcinoma showed apical expression of CD13, resembling to some extent CD1 $3^{\text {can }}$. Sensitivity and specificity were $96.2 \%$ and $97.0 \%$, respectively, for CD1 $3^{\text {can }}, 81.1 \%$ and $100 \%$ for p-CEA ${ }^{\text {can }}, 62.3 \%$ and $100 \%$, for CD10 10 , $54.7 \%$ and $99.9 \%$ for HepPar 1 , and $32.1 \%$ and $100 \%$ for AFP.

Conclusions: These results show that $\mathrm{CD} 13^{\text {can }}$ is more sensitive in differentiating between HCC and nonHCC than CD10 ${ }^{\text {can }}$, p-CEA ${ }^{\text {can }}$, HepParl, and AFP.
$\mathrm{P}$ rimary and metastatic malignant tumours of the liver may demonstrate a wide variety of histological patterns and the surgical pathologist is often challenged with biopsy specimens that yield only a small fraction of the liver mass lesion. Hepatocellular carcinomas (HCCs) and cholangiocarcinomas or metastatic tumours can often be differentiated using routine light microscopy, but it may be difficult to make this distinction, and special stains are needed. Special stains of diagnostic value in differentiating between HCC and non-HCC neoplasms include immunostaining with polyclonal antibodies crossreacting with biliary glycoprotein 1 (p-CEA), ${ }^{1-13}$ immunostaining with antibodies directed against $\alpha$ fetoprotein (AFP), ${ }^{1-367} \mathrm{CDl0},{ }^{9-14}$ and HepParl, ${ }^{6}{ }^{10-16}$ and detection of albumin mRNA by in situ hybridisation. ${ }^{17 \text { 17-20 }}$ Immunostaining for p-CEA and CD10 shows a characteristic canalicular pattern, with a sensitivity ranging from $50 \%$ to $90 \%$ and a specificity of almost $100 \%$. Other antibodies and antigens have been tested, but these have proved to be less useful or have not yet been confirmed, including $\alpha \mathrm{l}$ antitrypsin, ${ }^{21}$ monoclonal antibodies directed against carcinoembryonic antigen, ${ }^{368}$ various cytokeratins, $^{1262022}$ epithelial membrane antigen, ${ }^{8}$ erythropoiesis associated antigen, ${ }^{21}$ factor XIII, ${ }^{2}$ and $\mathrm{p}^{2} 8^{\mathrm{GANK}}{ }^{14}$

\section{"CD13 might be useful as an additional marker in differentiating between hepatocellular carcinoma and non-hepatocellular neoplasms"}

It was shown recently that aminopeptidase $\mathrm{N}(\mathrm{CD} 13)$ is expressed in both normal and neoplastic liver tissue, where it exhibits a canalicular distribution pattern $\left(\mathrm{CD}^{3} 3^{\mathrm{can}}\right)$ similar to that seen for p-CEA and CD10923; thus, CD13 might be useful as an additional marker in differentiating between HCC and non-hepatocellular neoplasms. The aim of our retrospective study was to investigate the sensitivity, specificity, and spatial distribution of CD13 in HCC and non-HCC compared with immunostaining for p-CEA ${ }^{\mathrm{can}}, \mathrm{CD}_{10} 0^{\mathrm{can}}$, HepParl, and AFP.

\section{MATERIALS AND METHODS}

\section{Case selection}

Fifty three HCC specimens, comprising 50 liver biopsies and three resection specimens, were retrieved from the archive of the department of pathology, Otto-von-Guericke-University, Germany. All cases were reviewed before study inclusion. In addition, most cases had been discussed during weekly clinicopathological conferences. Cases with ambiguous clinical or histological diagnostic features were not included in our study. The diagnosis of HCC was based on cytological, histological, and clinical criteria. Several clinical criteria supported the diagnosis of HCC, including evidence of a chronic diffuse liver disease with either liver fibrosis or cirrhosis, raised serum AFP values, and absence of an extrahepatic primary tumour. The specimens were obtained from 50 patients with an average age of 70.1 years (range, 49-95), 40 of whom were male and 10 were female (male to female ratio, $4: 1)$. The HCCs were categorised into well $(G 1)$, moderately (G2), or poorly (G3) differentiated types, corresponding to Edmondson's grades I/II, III, or IV, respectively. ${ }^{24}{ }^{25}$ As a control group (non-HCC), we selected 32 biopsy specimens of liver metastases from 32 patients; the primary site of the malignant tumour was confirmed clinically and/or histologically (table 1). The average age of the patients in the non-HCC group was 68.2 years (range, 4088 ) with 20 male and 12 female patients (male to female ratio, $1.6: 1)$.

Abbreviations: AFP, $\alpha$ fetoprotein; can, canalicular pattern; $C D 13$, aminopeptidase N; DAB, 3,3-diaminobenzidinetetrahydrochloride; $\mathrm{HCC}$, hepatocellular carcinoma; $\mathrm{p}$-CEA, antibody that crossreacts with biliary glycoprotein I; RT, room temperature 


\begin{tabular}{|c|c|c|c|c|}
\hline No. & $\begin{array}{l}\text { Age } \\
\text { (years) }\end{array}$ & Sex & Diagnosis & Primary \\
\hline 1 & 75 & M & Small cell carcinoma & Lung \\
\hline 2 & 81 & M & Small cell carcinoma & Lung \\
\hline 3 & 47 & M & Small cell carcinoma & Lung \\
\hline 4 & 75 & $\mathrm{~F}$ & Poorly differentiated non-small cell cancer & Lung \\
\hline 5 & 72 & M & Poorly differentiated non-small cell cancer & Lung \\
\hline 6 & 76 & M & Poorly differentiated non-small cell cancer & Lung \\
\hline 7 & 46 & $\mathrm{~F}$ & Poorly differentiated invasive ductal carcinoma & Breast \\
\hline 8 & 82 & $\mathrm{~F}$ & Poorly differentiated invasive ductal carcinoma & Breast \\
\hline 9 & 64 & $\mathrm{~F}$ & Undifferentiated carcinoma NOS & Breast \\
\hline 10 & 65 & $\mathrm{~F}$ & Moderately differentiated invasive ductal carcinoma & Breast \\
\hline 11 & 79 & $\mathrm{~F}$ & Undifferentiated carcinoma NOS & Breast \\
\hline 12 & 61 & M & Undifferentiated carcinoma NOS & Oesophagus \\
\hline 13 & 43 & M & Undifferentiated carcinoma NOS & Oesophagus \\
\hline 14 & 82 & M & Poorly differentiated neuroendocrine carcinoma & Stomach \\
\hline 15 & 68 & M & Poorly differentiated adenocarcinoma, intestinal type & Stomach \\
\hline 16 & 88 & M & Undifferentiated carcinoma NOS & Stomach \\
\hline 17 & 79 & $\mathrm{~F}$ & Poorly differentiated adenocarcinoma & Colon \\
\hline 18 & 71 & M & Poorly differentiated cholangiocarcinoma & Liver \\
\hline 19 & 64 & M & Poorly differentiated cholangiocarcinoma & Liver \\
\hline 20 & 65 & M & Poorly differentiated adenocarcinoma NOS & Extrahepatic bile ducts \\
\hline 21 & 71 & M & Poorly differentiated neuroendocrine carcinoma & Pancreas \\
\hline 22 & 57 & M & Poorly differentiated neuroendocrine carcinoma & Pancreas \\
\hline 23 & 82 & M & Undifferentiated carcinoma NOS & Pancreas \\
\hline 24 & 63 & M & Undifferentiated carcinoma NOS & Pancreas \\
\hline 25 & 73 & M & Poorly differentiated ductal adenocarcinoma & Pancreas \\
\hline 26 & 58 & M & Poorly differentiated ductal adenocarcinoma & Pancreas \\
\hline 27 & 75 & $\mathrm{~F}$ & Poorly differentiated serous papillary carcinoma & Ovary \\
\hline 28 & 40 & $\mathrm{~F}$ & Poorly differentiated squamous cell carcinoma & Cervix \\
\hline 29 & 63 & $\mathrm{~F}$ & Poorly differentiated squamous cell carcinoma & Cervix \\
\hline 30 & 71 & M & Well differentiated neuroendocrine carcinoma & CUP \\
\hline 31 & 66 & $\mathrm{~F}$ & Poorly differentiated neuroendocrine carcinoma & CUP \\
\hline 32 & 70 & $\mathrm{~F}$ & Small cell (neuroendocrine) carcinoma & CUP \\
\hline
\end{tabular}

\section{Specimen processing}

All biopsy and resection specimens were fixed in $10 \%$ buffered formalin and embedded in paraffin wax. Dewaxed serial sections were stained with haematoxylin and eosin, periodic acid Schiff with and without diastase pretreatment, and reticulin stain. Serial sections were cut at $3 \mu \mathrm{m}$ and placed on Superfrost Plus glass slides.

\section{Materials}

Immunostaining was performed with monoclonal antibodies directed against CD10 (clone 56C6), CD13 (clone 38C12; both Novocastra Laboratories, distributed by Medac $\mathrm{GmbH}$, Wedel, Germany), and HepParl (Dako, Glostrup, Denmark), and with polyclonal antibodies directed against AFP (Dako), and carcinoembryonic antigen (which crossreacts with biliary glycoprotein 1; p-CEA; Quartett, Berlin, Germany).

\section{Immunohistochemistry}

For immunostaining, sections were dewaxed in xylene and rehydrated in an alcohol series. Endogenous biotin was blocked using the endogenous biotin blocking kit from Ventana (Strasbourg, France). Immunostaining with p-CEA required blockade of endogenous peroxidase with $3 \% \mathrm{H}_{2} \mathrm{O}_{2}$ for 15 minutes at room temperature (RT) before the addition of p-CEA for one hour at $37^{\circ} \mathrm{C}(1 / 500$ dilution) in a moist chamber. This was followed by incubation with biotinylated secondary antibody and the streptavidin-peroxidase complex, each for 15 minutes at RT. Between steps, the sections were washed in Tris buffered saline. Immunostaining for CD10 and HepParl required pretreatment with 1mM EDTA (pH 8.0, 20 minutes, $450 \mathrm{~W}$ microwave oven), and for CD13 required pretreatment with $10 \mathrm{mM}$ sodium citrate $(\mathrm{pH} 6.0$, $3 \times 10$ minutes, $600 \mathrm{~W}$ microwave oven). Sections were incubated with anti-CD10 (1/25 dilution) and anti-CD13 (1/ 50 dilution) for one hour at $37^{\circ} \mathrm{C}$ in a moist chamber, followed by incubation with biotinylated antimouse IgG/ antirabbit IgG (1/200 dilution; Vector Laboratories; distributed by Camon, Wiesbaden, Germany) and ABC alkaline phosphatase reagent, each for 30 minutes at RT. Immunoreactions were visualised with the avidin-biotin complex method, applying a Vectastain $\mathrm{ABC}$ alkaline phosphatase kit (distributed by Camon) or an Ultratech horseradish peroxidase streptavidin-biotin universal detection system (Immunotech, Marseilles, France). Fast red and 3,3-diaminobenzidinetetrahydrochloride (DAB), respectively, served as chromogens.

Immunostaining with HepParl ( $1 / 50$ dilution) was performed using the Ventana enhanced alkaline phosphatase red detection kit and the Ventana Nexus immunostainer. The primary antibody was incubated for 30 minutes at $37^{\circ} \mathrm{C}$. The biotinylated secondary antibody and the alkaline phosphatase-streptavidin conjugate were applied according to the manufacturer's instructions. Fast red served as chromogen.

Immunostaining with anti-AFP ( $1 / 100$ dilution) was performed using the Ventana Basic DAB detection kit and the Ventana Nexus immunostainer. Endogenous peroxidase was blocked for four minutes at $37^{\circ} \mathrm{C}$, according to the manufacturer's instructions. The primary antibody was incubated for 26 minutes at $37^{\circ} \mathrm{C}$. The biotinylated secondary antibody, the avidin-horseradish peroxidase conjugate, and the basic DAB solution were applied according to the manufacturer's instructions. The reaction was enhanced with copper sulfate solution (four minutes at $37^{\circ} \mathrm{C}$ ).

All specimens were counterstained with haematoxylin. Primary antibodies were omitted for negative controls and tissue specimens recommended by the manufacturers were used as positive controls. 

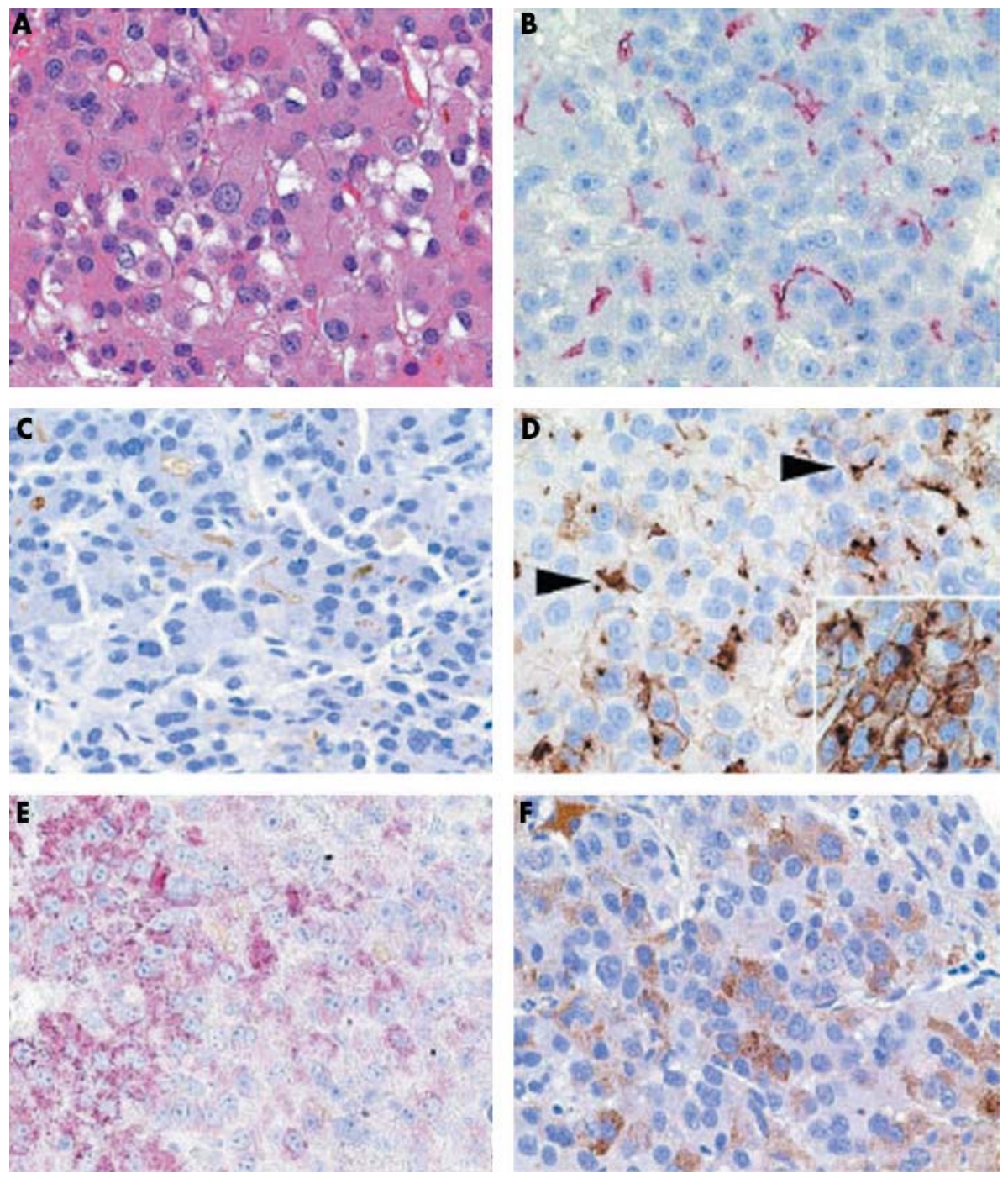

Figure 1 (A) A moderately differentiated hepatocellular carcinoma showing canalicular expression of (B) CD13 (aminopeptidase N), (C) p-CEA (antibody that crossreacts with biliary glycoprotein I), and (D, arrowheads) CD10, and cytoplasmic staining with (E) HepParl and (F) anti-AFP

( $\alpha$ fetoprotein). ( $D$, insert) Additional strong membranous expression was seen for CD10. (A) Haematoxylin and eosin; (B) anti-CD13, (C) anti-p-CEA, (D) anti-CD 10, (E) HepPar 1, and (F) anti-AFP, all with haematoxylin counterstain; original magnifications, $\times 400$.

\section{RESULTS}

\section{Hepatocellular carcinomas}

CD13 was detected in 51 of the 53 HCC specimens. Two CD13 immunostaining patterns were observed: cytoplasmic and cell membrane. Cell membrane staining was further divided into canalicular (delineating bile canaliculi) and noncanalicular patterns. A canalicular pattern was found in 51 specimens-all 14 well differentiated, 34 of 35 moderately differentiated, and three of four poorly differentiated HCCs (fig 1). Nine HCC specimens showed a cytoplasmic staining pattern and six non-canalicular staining of the cell membrane. Cytoplasmic staining of less than $10 \%$ of the tumour cells was found in six biopsies, staining of $10-50 \%$ of the tumour cells in three biopsies, and staining of greater than $50 \%$ of the tumour cells was not seen. Simultaneous cytoplasmic and cell membrane staining was found in nine of the 53 biopsy specimens. Non-neoplastic liver tissue showed a canalicular staining pattern and apical membranous staining of bile ducts (fig 2 ).

Immunostaining with p-CEA was found in 43 of 53 HCC specimens. The staining pattern was similar to that seen for CD13-cytoplasmic, canalicular, and non-canalicular (fig 1). A canalicular pattern was found in 43 specimens, a noncanalicular pattern in 13 specimens, and cytoplasmic staining was found in 13 specimens. Overall, the extent of the canalicular staining pattern was decreased in poorly differ- entiated HCCs; 10 of 14 well differentiated HCCs, 31 of 35 moderately differentiated HCCs, and two of four poorly differentiated HCCs showed a canalicular pattern. Nonneoplastic liver parenchyma showed canalicular immunostaining (fig 2).

CD10 was detected in 42 of the 53 HCC specimens. The staining pattern was similar to that seen for CD13 and pCEA-cytoplasmic, canalicular, and non-canalicular (fig 1). A canalicular pattern was found in 33 specimens and the prevalence of canalicular staining correlated with the histological grade: 12 of 14 G1 HCCs, 19 of 35 G2 HCCs, and two of four G3 HCCs showed canalicular staining. Thirty HCC specimens had a cytoplasmic staining pattern and eight had non-canalicular staining of the cell membrane. Cytoplasmic staining of less than $10 \%$ of the tumour cells was found in 14 biopsies, staining of $10-50 \%$ in eight biopsies, and staining of greater than 50\% in eight biopsies. Simultaneous cytoplasmic and cell membrane staining was found in 21 of the 53 biopsy specimens. There was no significant difference in the overall expression of CD10 between well and moderately differentiated HCCs. Nonneoplastic liver tissue showed a canalicular staining pattern in the parenchyma and apical membranous staining of bile ducts (fig 2).

Immunostaining with HepParl was found in 29 of 53 HCC specimens; HepParl stained the cytoplasm only (fig 1). The 

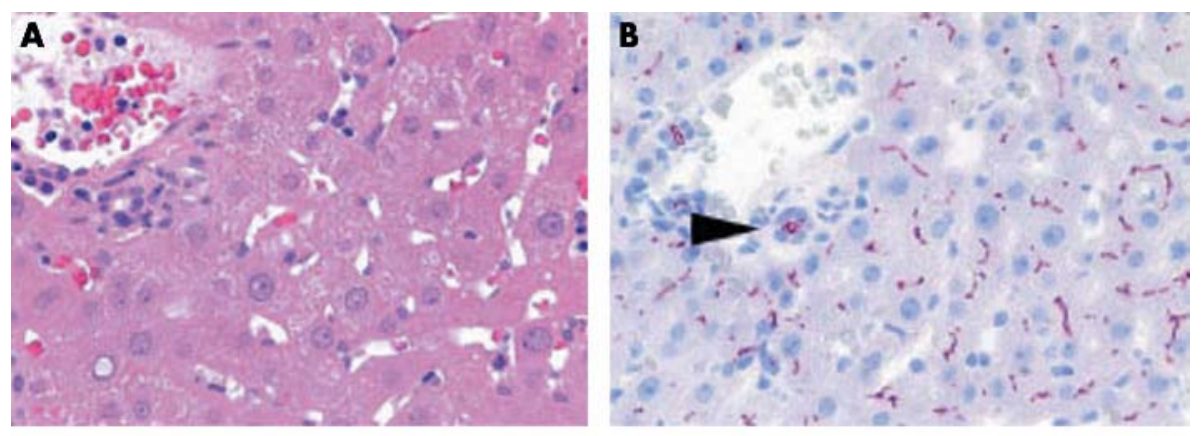

Figure 2 (A) Non-neoplastic liver tissue showing canalicular immunostaining with (B) anti-CD13 (aminopeptidase N), (C) anti-p-CEA (antibody that crossreacts with biliary glycoprotein I), and (D) anti-CD10. Bile ducts express $C D 10$ and $C D 13$ at the apical membrane $(B$ and $D$; arrowheads). (A) Haematoxylin and eosin; (B) anti-CD13, (C) anti-p-CEA, and (D) anti-CD10, all with

haematoxylin counterstain; original magnifications, $\times 400$.
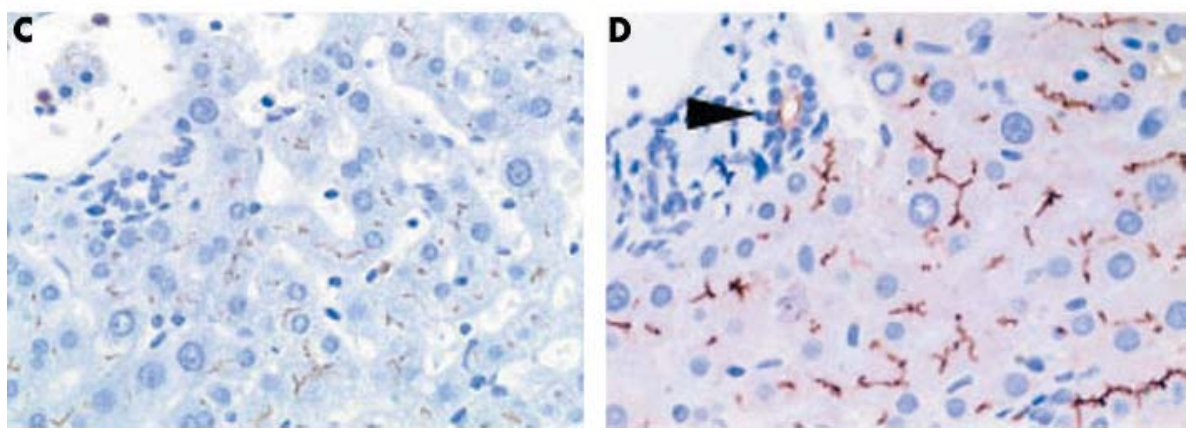

prevalence of HepParl immunostaining correlated inversely with the histological grade of the HCCs; HepParl-staining was found in 10 of 14 well differentiated, 19 of 35 moderately differentiated, and none of the four poorly differentiated HCCs.

Immunostaining for AFP was found in 17 of 53 HCC specimens; AFP was found both in the cytoplasm and occasionally at the cell membrane (fig 1). The prevalence of AFP correlated with the histological grade of the HCCs; AFP was found in two of 14 G1, 12 of 35 G2, and three of four G3 HCCs.

Figure 3 summarises the distribution pattern of all five immunohistochemical markers (CD13, p-CEA, and CDl0canalicular pattern only; HepParl and AFP-cytoplasm and/ or cell membrane). Only two cases showed no $\mathrm{CD}^{\mathrm{can}}$ immunostaining: one specimen stained for none of the immunohistochemical markers studied, although follow up biopsies clearly showed the HCC nature of the specimen. The second specimen showed canalicular immunostaining for CDI0 and p-CEA, but lacked CDI3 immunoreactivity. Interestingly, the bile canaliculi of HCCs were more often immunoreactive for $\mathrm{CD} 13$ than for p-CEA or CDl0 (fig 3).

\section{Non-hepatocellular carcinomas}

Among the non-HCCs, CD13 was present in the cytoplasm of one specimen-a poorly differentiated cholangiocarcinoma. Apical membranous staining was found in two cases-a metastasis of a poorly differentiated serous papillary carcinoma of the ovary and a poorly differentiated cholangiocarcinoma (fig 4). In the latter, CDl3 immunostaining resembled canalicular staining of HCC. However, a desmoplastic stroma was present and all other HCC markers were negative $\left(\mathrm{CDI}^{\mathrm{can}}, \mathrm{p}-\mathrm{CEA}^{\mathrm{can}}\right.$, HepParl, and AFP).

p-CEA staining was positive in 17 of the 32 specimens: staining was cytoplasmic only in 10, membranous only in one, and both cytoplasmic and membranous in six specimens. CDlo was present in the cytoplasm and at the cell membrane. Six of the 32 biopsy specimens showed cytoplasmic staining and four showed both cytoplasmic and cell membrane staining. In seven biopsy specimens, less than $10 \%$ of the tumour cells were immunoreactive, and more than $50 \%$ were immunoreactive in three. A canalicular staining pattern for CDI0 and p-CEA was not detected in the non-HCC specimens.

HepParl immunostaining was found in only one specimen obtained from a poorly differentiated cholangiocarcinoma (fig 4). AFP staining was negative in all of the non-HCCs.

The sensitivity and specificity were calculated as $96.2 \%$ and $97.0 \%$, respectively, for $\mathrm{CD} 13^{\mathrm{can}}, 81.1 \%$ and $100 \%$ for $\mathrm{p}$ $\mathrm{CEA}^{\mathrm{can}}, 62.3 \%$ and $100 \%$, for $\mathrm{CD}^{\mathrm{can}}, 54.7 \%$ and $99.9 \%$ for HepParl, and $32.1 \%$ and $100 \%$ for AFP.

Table 2 summarises the immunostaining results for HCC and non-HCC.

\section{DISCUSSION}

Aminopeptidase N (CDI3, APN) is a zinc dependent, cell membrane metallopeptidase, which has been shown to participate in the postsecretory processing of neuropeptides and peptide hormones. It is widely distributed and has been found in various cell types of organs and tissues, including

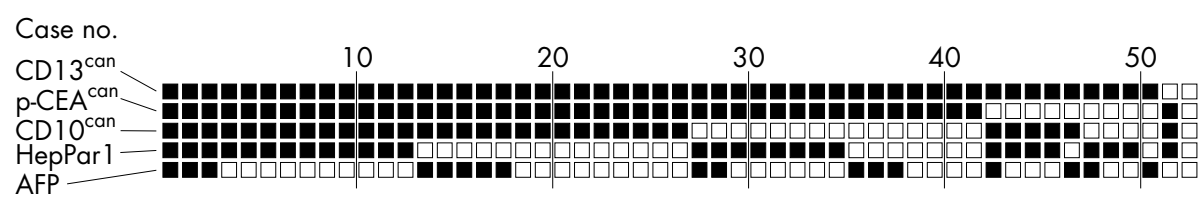

Figure 3 Immunohistochemical expression profile of 53 hepatocellular carcinomas (HCCs) for CD13 (aminopeptidase N), p-CEA (antibody that crossreacts with biliary glycoprotein I), CD10, HepParl, and AFP ( $\alpha$ fetoprotein). Each column represents an individual biopsy sample from an HCC. Black squares denote positive canalicular immunostaining for CD13 (CD13 $\left.{ }^{\text {can }}\right)$, p-CEA ( $\mathrm{p}-\mathrm{CEA}^{\text {can }}$ ), and CD10 (CD10 ${ }^{\text {can }}$ ), and cytoplasmic or membranous immunostaining with HepParl and for AFP, respectively; open squares denote no immunoreactivity in the tumour cells. 

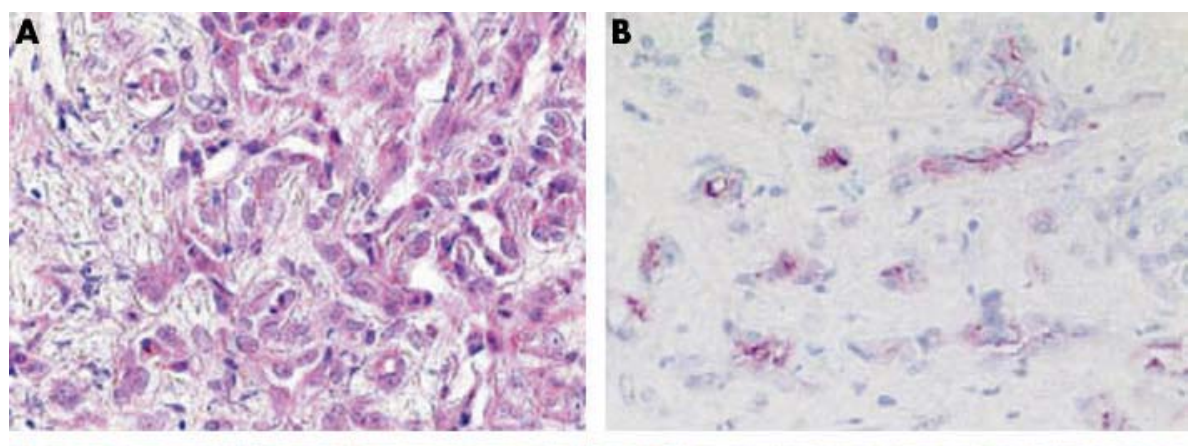

Figure 4 (A) A cholangiocarcinoma shows apical membranous expression of (B) CD13, resembling to some extent canalicular expression of hepatocytes. (D) Cytoplasmic HepPar 1 immunostaining was found in another (C) cholangiocarcinoma. (A, C) Haematoxylin and eosin; (B) antiCD13, (D) HepParl, both with haematoxylin counterstain; original magnifications, $\times 400$.
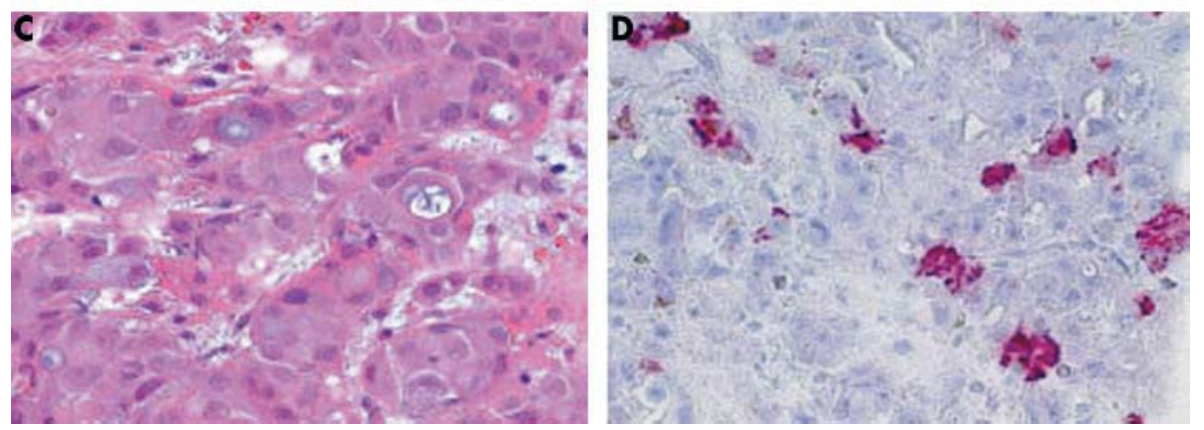

benign and malignant tumours. The expression and putative pathophysiological role of $\mathrm{CD} 13$ has been studied in a variety of malignant tumours. ${ }^{26}{ }^{27}$ The expression of CD13 has been linked to tumour cell proliferation, degradation of extracellular matrix, and metastatic behaviour. ${ }^{27-32}$ Almost all of these biological effects were attributed to the ectopeptidase activity.

Recently, it was shown that CD13 is also expressed by HCCs. ${ }^{23}$ Interestingly, CD13 mRNA showed no significant differences between non-tumorous liver and HCC, whereas $\mathrm{CD} 13$ protein values were slightly increased. ${ }^{23} \mathrm{CD} 13$ may have a pathophysiological effect on hepatocarcinogenesis by cleaving regulatory peptides and peptide hormones. However, CD13 may have another role in HCC, which is unique to the liver-that is, the formation of bile canaliculi and production and secretion of bile acids. Previously, we found CD13 positive bile canaliculi in the fetal liver, focal nodular hyperplasia, non-tumorous liver, and HCC. ${ }^{23}$ This reflects its ubiquitous expression and its close association with the formation and function of bile canaliculi. ${ }^{33} \mathrm{CD} 13$ positive bile canaliculi have been detected as early as 16 to 18 weeks of gestation, ${ }^{23}$ underscoring the role of this molecule in morphogenesis. Expression of $\mathrm{CDl} 3$ is maintained at a constant level during liver regeneration. ${ }^{34}$ Thus, expression of $\mathrm{CD} 13$ seems to be required in various proliferation and differentiation states of the liver, which makes it an attractive diagnostic marker for surgical pathology.

"CD13 may have a role in hepatocellular carcinoma that is unique to the liver-that is, the formation of bile canaliculi and production and secretion of bile acids"

Because CD13 shows a specific canalicular staining pattern, ${ }^{23}$ similar to that seen for $\mathrm{p}-\mathrm{CEA}^{\mathrm{can}}$ and $\mathrm{CD} 10^{\mathrm{can}}$, our present study aimed to investigate the putative use of $\mathrm{CD}_{1} 3^{\mathrm{can}}$ in differentiating HCCs from non-HCCs.

In our current series, both HCCs and non-HCCs expressed CD13 in the cytoplasm, at the cell membrane, or both, and the detection of CD13 itself was of no use in differentiating HCCs from non-HCCs. However, only non-neoplastic liver tissue and HCC showed a characteristic canalicular staining pattern, similar to that seen for p-CEA and CD10; this pattern was considered to be specific for HCCs and yielded a

Table 2 Immunostaining of hepatocellular (HCC) and non-hepatocellular (non-HCC) carcinomas

\begin{tabular}{|c|c|c|c|c|c|}
\hline & \multicolumn{4}{|l|}{$\mathrm{HCC}$} & \multirow{2}{*}{$\frac{\text { Non-HCC }}{n / n(\%)}$} \\
\hline & Total n/n (\%) & G1 n/n (\%) & G2 n/n (\%) & G3 n/n (\%) & \\
\hline \multicolumn{6}{|l|}{ CD13 } \\
\hline Cytoplasm & $9 / 53(17.0)$ & $0 / 14$ & $8 / 35$ (22.9) & $1 / 4(25.0)$ & $1 / 32(3.1)$ \\
\hline Cell membrane (non-canalicular) & $6 / 53(11.3)$ & $3 / 14(21.4)$ & $2 / 35(5.7)$ & $1 / 4(25.0)$ & $1 / 32(3.1)$ \\
\hline Cell membrane (canalicular) & $51 / 53(96.2)$ & $14 / 14$ & $34 / 35(97.1)$ & $3 / 4(75.0)$ & $1 / 32(3.1)$ \\
\hline \multicolumn{6}{|l|}{ p-CEA } \\
\hline Cytoplasm & $13 / 53(24.5)$ & $2 / 14(14.3)$ & $9 / 35(25.7)$ & $2 / 4(50.0)$ & $16 / 32(50.0)$ \\
\hline Cell membrane (non-canalicular) & $14 / 53(26.4)$ & $4 / 14(28.6)$ & $8 / 35(22.9)$ & $2 / 4(50.0)$ & $7 / 32(21.9)$ \\
\hline Cell membrane (canalicular) & $43 / 53(81.1)$ & $10 / 14(71.4)$ & $31 / 35(88.6)$ & $2 / 4(50.0)$ & $0 / 32$ \\
\hline \multicolumn{6}{|l|}{ CDIO } \\
\hline Cytoplasm & $30 / 53(56.6)$ & 10/14 (71.4) & $18 / 35(51.4)$ & $2 / 4(50.0)$ & $10 / 32(31.3)$ \\
\hline Cell membrane (non-canalicular) & $8 / 53(15.1)$ & $4 / 14(28.6)$ & $3 / 35(8.6)$ & $1 / 4(25.0)$ & $4 / 32(12.5)$ \\
\hline Cell membrane (canalicular) & $33 / 53(62.3)$ & $12 / 14(85.7)$ & $19 / 35(54.3)$ & $2 / 4(50.0)$ & $0 / 32$ \\
\hline HepParl & $29 / 53(54.7)$ & $10 / 14(71.4)$ & $19 / 35(54.3)$ & $0 / 4$ & $1 / 32(3.1)$ \\
\hline AFP & $17 / 53(32.1)$ & $2 / 14(14.3)$ & $12 / 35(34.3)$ & $3 / 4(75.0)$ & $0 / 32$ \\
\hline
\end{tabular}


Table 3 Summary of recent studies investigating the sensitivity and specificity of canalicular immunostaining for CD10 and pCEA

\begin{tabular}{|c|c|c|c|c|}
\hline \multirow[b]{2}{*}{ First author } & \multicolumn{2}{|l|}{ CD $10^{\text {can }}$} & \multicolumn{2}{|l|}{ p-CEA ${ }^{\text {can }}$} \\
\hline & HCC n/n (\%) & Non-HCC $n / n(\%)$ & HCC $n / n(\%)$ & Non-HCC $n / n(\%)$ \\
\hline Borscheri ${ }^{9}$ & $43 / 63(68.3 \%)$ & $0 / 25(0 \%)$ & $60 / 63(95.2 \%)$ & $0 / 25(0 \%)$ \\
\hline Chu" & $50 / 96(52.1 \%)$ & ND & $73 / 96$ (76.0\%) & ND \\
\hline $\mathrm{Lau}^{13}$ & $17 / 42(40.5 \%)$ & $0 / 65(0 \%)$ & $29 / 42(69.0 \%)$ & $0 / 56(0 \%)$ \\
\hline Lee $^{15}$ & $21 / 75(28.0 \%)$ & $0 / 399(0 \%)$ & ND & ND \\
\hline $\operatorname{Lin}^{35}$ & $19 / 22(86.4 \%)$ & $0 / 23(0 \%)$ & ND & ND \\
\hline Morrison $^{12}$ & $13 / 25$ (52.0\%) & $0 / 75(0 \%)$ & $24 / 25$ (96.0\%) & $2 / 75(2.7 \%)$ \\
\hline Saad $^{10}$ & $23 / 30(76.7 \%)$ & $0 / 30(0 \%)$ & $24 / 30(80.0 \%)$ & $0 / 30(0 \%)$ \\
\hline$X_{i a o^{36}}$ & $9 / 15(60.0 \%)$ & $0 / 19(0 \%)$ & $15 / 15(100 \%)$ & $0 / 19(0 \%)$ \\
\hline Our present study & $33 / 53(62.3 \%)$ & $0 / 33(0 \%)$ & $43 / 53(81.1 \%)$ & $0 / 33(0 \%)$ \\
\hline Summary & $228 / 421(54.2 \%)$ & $0 / 669(0 \%)$ & $268 / 324$ (82.7\%) & $2 / 238(0.8 \%)$ \\
\hline
\end{tabular}

HCC, hepatocellular carcinoma; ND, not determined; $\mathrm{p}$-CEA, antibody that crossreacts with biliary glycoprotein I.

sensitivity of $96.2 \%$ and a specificity of $97.0 \%$ in our series. To evaluate further the diagnostic use of $\mathrm{CD} 13^{\mathrm{can}}$ in differentiating HCC from non-HCC, we compared the sensitivity and specificity of $\mathrm{CD}_{3}{ }^{\mathrm{can}}$ with p-CEA ${ }^{\mathrm{can}}, \mathrm{CDI}^{\mathrm{can}}$, HepParl, and AFP-markers that have proved their diagnostic usefulness in surgical pathology..$^{1-3} 67^{19-21}$ In our series, the sensitivity of $\mathrm{CD}_{1} 3^{\mathrm{can}}$ was greater than that of $\mathrm{p}-\mathrm{CEA}^{\mathrm{can}}, \mathrm{CD}^{\mathrm{can}}$, HepParl, and AFP. Previous reports have described a canalicular staining pattern for p-CEA in $24-90 \%$ of cases $^{1-46}{ }^{7}$ and for CD10 in $28-86 \%$ of cases $^{1-4} 67$; our figures were $81.1 \%$ and $62.3 \%$, respectively. Thus, our values for canalicular immunostaining of anti-p-CEA and anti-CD10 are within the range of previous observations. Table 3 summarises the results of more recent studies investigating the diagnostic use of $\mathrm{CD}_{10} 0^{\mathrm{can}}$ and p-CEA ${ }^{\mathrm{can}}$. Table 3 shows that the sensitivity of $\mathrm{CDIO}^{\mathrm{can}}$ is much lower than that of $\mathrm{p}$ $\mathrm{CEA}^{\mathrm{can}}$. However, as shown in our present study, the difference between p-CEA ${ }^{\text {can }}$ and $\mathrm{CD}_{3} 3^{\mathrm{can}}$ is less pronounced, although unlike p-CEA and CDI0, CDI3 stained the cytoplasm of tumour cells less often, facilitating the recognition of even low numbers of bile canaliculi, which we consider to be an advantage of $\mathrm{CD13}$, particularly in poorly differentiated HCCs.

CD13 was also expressed in bile ducts and in a cholangiocarcinoma, here resembling canalicular immunostaining. However, cholangiocarcinomas have an abundant desmoplastic stroma that aids in differentiating them from HCC in most cases.

HepParl is a monoclonal antibody that recognises a mitochondrial antigen of hepatocytes. ${ }^{637}$ In the past few years, several studies have investigated the sensitivity and specificity of HepParl, ${ }^{10-1638}$ and these were shown to range from $75 \%$ to $100 \%$ and from $66 \%$ to $100 \%$, respectively. In our series, we found HepParl staining in only 29 of the 53 (54.7\%) HCCs. This rather low sensitivity may be a sampling error, because most of our specimens were biopsies: HepParl staining is not homogeneous and only eight specimens showed HepParl staining in more than $50 \%$ of the tumour cells, and in most of our cases HepParl stained less than 50\% of the tumour cells. Furthermore, in contrast to CD13, p-CEA, and CDI0, immunostaining with HepParl does not show a hepatocyte specific staining pattern. Between $44 \%$ and $47 \%$ of gastric cancers react with HepParl, ${ }^{16}{ }^{38}$ and differentiating poorly differentiated gastric cancer from HCC using HepParl immunostaining only can be difficult at times. HepParl also occasionally stains cholangiocarcinomas (our present study $)^{11}{ }^{15} 38$, and pancreatic, colon, lung, adrenal, neuroendocrine, ovarian, and endocervical cancers. ${ }^{11}{ }^{15} 38$ Thus, although HepParl frequently reacts with HCCs, it should be used cautiously and in conjunction with a panel of other antibodies, as recently stated by Fan et al. ${ }^{38}$

\section{Take home messages}

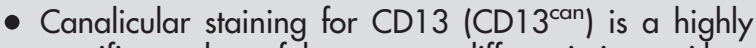
specific marker of hepatocyte differentiation, with a sensitivity greater than that of $\mathrm{p}-\mathrm{CE} \mathrm{A}^{\mathrm{can}}, \mathrm{CD} 10^{\text {can }}$, HepPar 1, and $\alpha$ fetoprotein

- Although $\mathrm{CD} 13^{\text {can }}$ does not differentiate between benign and malignant lesions, it is clearly useful for differentiating hepatocellular carcinoma (HCC) from non-HCC lesions

- Further studies are needed to determine whether CD13 could replace $\mathrm{p}-\mathrm{CEA}$ and $\mathrm{CD} 10$ in the diagnostic hepatopathology of HCCs and liver metastases

In our series, the sensitivity of $\mathrm{CD} 13^{\mathrm{can}}, \mathrm{p}-\mathrm{CEA}^{\mathrm{can}}, \mathrm{CD} 10^{\mathrm{can}}$, and HepParl was superior to that of AFP; only 16 of the 53 HCCs expressed AFP. Previous studies have shown that between $17 \%$ and $62 \%$ of HCCs show immunostaining for AFP, ${ }^{1-3} 67$ and AFP immunostaining in our HCC specimens was within this range.

Sensitivity and specificity are influenced by many variables, with sampling being the most important. Biopsy specimens often provide only a small fraction of the tumour, so that a lack of immunostaining may simply be the result of inadequate sampling (see above). All five markers tested here are subject to sampling errors. Thus, to reduce sampling errors, using a battery of different markers has become common practice in cases where the histological diagnosis is not readily apparent from routine histochemical stains (for example, haematoxylin and eosin, periodic acid Schiff, and reticulin stain). By comparing the staining patterns of $\mathrm{CD} 13$, p-CEA, CDI0 can, HepParl, and AFP, we were able to show that only one specimen was negative for all five markers. A combination of $\mathrm{CDI}^{\mathrm{can}}$ (as the most sensitive marker for the presence of bile canaliculi), AFP (as a sensitive marker for poorly differentiated HCCs), and HepParl staining was diagnostic in $98.1 \%$ of our HCCs, whereas CD13 ${ }^{\text {can }}$ and AFP together were diagnostic in $96.2 \%$ of cases. Future studies are needed to determine whether $\mathrm{CD} 13$ has the potential to replace p-CEA and CD10 in the diagnostic hepatopathology of HCCs and liver metastases.

In summary, canalicular staining for $\mathrm{CD} 13$ is a highly specific marker of hepatocyte differentiation, with a sensitivity greater than that of $\mathrm{p}-\mathrm{CEA}^{\mathrm{can}}$ and $\mathrm{CD} 10^{\mathrm{can}}$. Although $\mathrm{CD}_{1} 3^{\mathrm{can}}$ does not differentiate between benign and malignant lesions, it is clearly of use in differentiating HCC from non-HCC. 
Authors' affiliations

C Röcken, J Licht, A Roessner, S Carl-McGrath, Department of Pathology, Otto-von-Guericke-University, Leipziger Str. 44, D-39120 Magdeburg, Germany

\section{REFERENCES}

1 D'Errico A, Baccarini P, Fiorentino, M, et al. Histogenesis of primary liver carcinomas: strengths and weaknesses of cytokeratin profile and albumin mRNA detection. Hum Pathol 1996;27:599-604.

2 Hurlimann J, Gardiol D. Immunohistochemistry in the differential diagnosis of liver carcinomas. Am J Surg Pathol 1991;15:280-8.

$3 \mathrm{Ma} \mathrm{CK}$, Zarbo RJ, Frierson HF Jr, et al. Comparative immunohistochemical study of primary and metastatic carcinomas of the liver. Am J Clin Pathol 1993;99:551-7.

4 Koelma IA, Nap M, Huitema S, et al. Hepatocellular carcinoma, adenoma, and focal nodular hyperplasia. Comparative histopathologic study with immunohistochemical parameters. Arch Pathol Lab Med 1986; 1 10: 1035-40.

5 Wong MA, Yazdi HM. Hepatocellular carcinoma versus carcinoma metastatic to the liver. Value of stains for carcinoembryonic antigen and naphthylamidase in fine needle aspiration biopsy material. Acta Cytol 1990;34:192-6.

6 Minervini MI, Demetris AJ, Lee RG, et al. Utilization of hepatocyte-specific antibody in the immunocytochemical evaluation of liver tumors. Mod Pathol 1997; 10:686-92

7 Oliveira AM, Erickson LA, Burgart $\sqcup$, et al. Differentiation of primary and metastatic clear cell tumors in the liver by in situ hybridization for albumin messenger RNA. Am J Surg Pathol 2000;24:177-82.

8 Christensen WN, Boitnott JK, Kuhajda FP. Immunoperoxidase staining as a diagnostic aid for hepatocellular carcinoma. Mod Pathol 1989;2:8-12.

9 Borscheri N, Roessner A, Röcken C. Canalicular immunostaining of neprilysin (CD10) as a diagnostic marker for hepatocellular carcinomas. Am J Surg Pathol 2001;25:1297-303.

10 Saad RS, Luckasevic TM, Noga CM, et al. Diagnostic value of HepParl, pCEA, CD10, and CD34 expression in separating hepatocellular carcinoma from metastatic carcinoma in fine-needle aspiration cytology. Diagn Cytopathol 2004;30:1-6.

11 Chu PG, Ishizawa S, Wu E, et al. Hepatocyte antigen as a marker of hepatocellular carcinoma: an immunohistochemical comparison to carcinoembryonic antigen, CD10, and alpha-fetoprotein. Am J Surg Pathol 2002;26:978-88.

12 Morrison C, Marsh W Jr. Frankel WL. A comparison of CD10 to pCEA, MOC 31 , and hepatocyte for the distinction of malignant tumors in the liver. Mod Pathol 2002;15:1279-87.

13 Lau SK, Prakash S, Geller SA, et al. Comparative immunohistochemical profile of hepatocellular carcinoma, cholangiocarcinoma, and metastatic adenocarcinoma. Hum Pathol 2002;33:1175-81.

14 Fu X, Tan L, Liu S, et al. A novel diagnostic marker, p28(GANK), distinguishes hepatocellular carcinoma from potential mimics. J Cancer Res Clin Oncol 2004; 130:514-20.

15 Lee HS, Kim WH, Kang GH. Hepatocyte expressions in hepatocellular carcinomas, gastrointestinal neoplasms, and non-neoplastic gastrointestinal mucosa: its role as a diagnostic marker. J Korean Med Sci 2003; 18:842-8

16 Siddiqui MT, Saboorian MH, Gokaslan ST, et al. Diagnostic utility of the HepParl antibody to differentiate hepatocellular carcinoma from metastatic carcinoma in fine-needle aspiration samples. Cancer 2002;96:49-52.

17 Murray GI, Paterson PJ, Ewen SW, et al. In situ hybridisation of albumin mRNA in normal liver and hepatocellular carcinoma with a digoxigenin labelled oligonucleotide probe. J Clin Pathol 1992;45:21-4.
18 Yamaguchi K, Nalesnik MA, Carr BI. In situ hybridization of albumin mRNA in normal liver and liver tumors: identification of hepatocellular origin. Virchows Arch B Cell Pathol Ind Mol Pathol 1993;64:361-5.

19 Krishna M, Lloyd RV, Batts KP. Detection of albumin messenger RNA in hepatic and extrahepatic neoplasms. A marker of hepatocellular differentiation. Am J Surg Pathol 1997;21:147-52.

20 Papotti M, Pacchioni D, Negro F, et al. Albumin gene expression in liver tumors: diagnostic interest in fine needle aspiration biopsies. Mod Pathol 1994:7:271-5.

21 Ganjei P, Nadji M, Albores-Saavedra J, et al. Histologic markers in primary and metastatic tumors of the liver. Cancer 1988;62:1994-8

22 Van Eyken P, Desmet VJ. Cytokeratins and the liver. Liver 1993;13:113-22.

23 Röcken C, Carl-McGrath S, Gräntzdörffer I, et al. Ectopeptidases are differentially expressed in hepatocellular carcinomas. Int J Oncol 2004; 24:487-95

24 International Working Party. Terminology of nodular hepatocellular lesions. Hepatology 1995;22:983-93.

25 MacSween RNM, Burt AD, Portmann BC, et al. Pathology of the liver. Edinburgh: Churchill Livingstone, 2002

26 Mechtersheimer G, Moller P. Expression of aminopeptidase N (CD13) in mesenchymal tumors. Am J Pathol 1990;137:1215-22.

27 Tokuhara T, Adachi M, Hashida H, et al. Neutral endopeptidase/CD10 and aminopeptidase N/CD13 gene expression as a prognostic factor in non-small cell lung cancer. Jpn J Thorac Cardiovasc Surg 2001;49:489-96.

28 Saiki I, Fujii $\mathrm{H}$, Yoneda J, et al. Role of aminopeptidase N (CDI3) in tumor-cell invasion and extracellular matrix degradation. Int J Cancer 1993;54:137-43.

29 Menrad A, Speicher D, Wacker J, et al. Biochemical and functional characterization of aminopeptidase $\mathrm{N}$ expressed by human melanoma cells. Cancer Res 1993;53:1450-5.

30 Kido A, Krueger S, Haeckel C, et al. Possible contribution of aminopeptidase $N$ (APN/CD13) to invasive potential enhanced by interleukin- 6 and soluble interleukin-6 receptor in human osteosarcoma cell lines. Clin Exp Metastasis 1999; 17:857-63.

31 Hashida $\mathrm{H}$, Takabayashi $\mathrm{A}$, Kanai $\mathrm{M}$, et al. Aminopeptidase $\mathrm{N}$ is involved in cell motility and angiogenesis: its clinical significance in human colon cancer. Gastroenterology 2002;122:376-86.

32 Ishii K, Usui S, Sugimura Y, et al. Inhibition of aminopeptidase N (AP-N) and urokinase-type plasminogen activator (UPA) by zinc suppresses the invasion activity in human urological cancer cells. Biol Pharm Bull $2001 ; 24: 226-30$.

33 Lian WN, Tsai JW, Yu PM, et al. Targeting of aminopeptidase $\mathrm{N}$ to bile canaliculi correlates with secretory activities of the developing canalicular domain. Hepatology 1999;30:748-60.

34 Bartles JR, Zhang LQ, Verheyen EM, et al. Decreases in the relative concentrations of specific hepatocyte plasma membrane proteins during liver regeneration: down-regulation or dilution? Dev Biol 1991;143:258-70.

35 Lin F, Abdallah H, Meschter S. Diagnostic utility of CD10 in differentiating hepatocellular carcinoma from metastatic carcinoma in fine-needle aspiration biopsy (FNAB) of the liver. Diagn Cytopathol 2004;30:92-7.

36 Xiao SY, Wang HL, Hart J, et al. cDNA arrays and immunohistochemistry identification of CD10/CALLA expression in hepatocellular carcinoma. Am J Pathol 2001;159:1415-21.

37 Leong AS, Sormunen RT, Tsui WM, et al. Hep Par 1 and selected antibodies in the immunohistological distinction of hepatocellular carcinoma from cholangiocarcinoma, combined tumours and metastatic carcinoma. Histopathology 1998;33:318-24.

38 Fan Z, van de Rijn M, Montgomery K, et al. Hep par 1 antibody stain for the differential diagnosis of hepatocellular carcinoma: 676 tumors tested using tissue microarrays and conventional tissue sections. Mod Pathol 2003;16:137-44. 